\title{
EDITORIAL
}

\section{Lower respiratory tract infections in the community: towards a more rational approach}

\author{
J. Dorca*, A. Torres**
}

Lower respiratory tract infections (LRTI), such as acute bronchitis, infectious exacerbation of chronic bronchitis and pneumonia, are frequent community-acquired infections affecting both the paediatric and adult population, and are one of the commonest respiratory illnesses observed in daily medical practice [1]. In most cases, LRTI cause a mild clinical picture, usually managed by general practitioners. Only a minority of episodes with a more severe presentation, or affecting patients with chronic diseases, are attended by hospital specialists. Because bacterial organisms are often involved or could potentially complicate the course of LRTI, antibiotic prescription on an empirical basis is the commonest approach, in spite of the benign and self-limited nature of the majority of episodes. Although there is a general acceptance that LRTI is the most frequent cause for antibiotic prescription in general medical practice, precise data about antibiotic regimens are scarce [2]. In addition, the number of drugs potentially active against respiratory pathogens has increased considerably and, consequently, the prescribed regimens may differ widely.

In this issue of the Journal, two excellent original studies coming from the same group provide fresh data about the current patterns of diagnostic management and antibiotic prescription in LRTI among general practitioners (GPs) of five European countries: France, Germany, Italy, Spain and the United Kingdom. In one of these studies, Huchon et al. [3] have concluded that antibiotics are excessively prescribed by European GPs; more than $80 \%$ of all LRTI, globally considered, are treated with antibiotics. This figure showed little variation as regards the different types of LRTI: community-acquired pneumonia, acute bronchitis, exacerbated chronic bronchitis, and "viral" respiratory tract infection. Even in this last group, $70 \%$ of cases were initially managed with antibiotics.

In contrast to the relative homogeneity in antibiotic prescription rates, the authors found substantial differences in the characteristics (nature) of antibiotic treatments. According to this, oral penicillins were first choice antibiotics in the UK and France, tetracyclines were preferred in Germany, macrolides were given in Spain, and parenteral third-generation cephalosporins were commonly prescribed in Italy. The pattern of prescription was relatively independent of the characteristics of LRTI. However, the authors could find no convincing reasons for these striking differences.

In an accompanying paper, also published in this issue of the Journal, WOODHEAD et al. [4] assessed the diagnostic management of LRTI by European GPs. These

*Correspondence: J. Dorca, Servei de Pneumologia, Hospital de Bellvitge, Feixa LLarga s/n, L'Hospitalet de LLobregat, Barcelona, Spain. **Servei de Pneumologia y Al.lérgia Respiratoria, Hospital Clinic, Barcelona, Spain. authors concluded that LRTI are, in most cases, managed only on clinical grounds. Therefore, diagnostic tests such as chest radiography, white blood cell (WBC) counts, or sputum Gram stain, were performed in only one third of cases.

Although the methodology used for collecting information in these two studies, (directly asking a sample of GPs what they remember about how they managed the last 3-4 LRTI they had attended) could be considered as being rather imprecise, they undoubtedly provide valuable information about daily habits in medical practice. In addition, these findings appear to agree with the data collected in other studies using different approaches to assess the patterns of antibiotic prescription for community-acquired pneumonia in Europe [3].

The scenario described in these studies is not satisfactory: antibiotic prescription for LRTI in general medicine appears to be excessive and, quite often, inappropriate. For example, most acute bronchitis, viral or bacterial, affecting young or middle-aged healthy individuals is in fact a benign and self-limited process that will not profit greatly from an antibiotic course. Even in exacerbated chronic bronchitis, only a minority of patients really suffer from a bacterial infection and will, eventually, improve with antibiotics [5]. By contrast, pneumonia is a potentially fatal disease if not adequately treated $[6,7]$. Because of the limited value of symptoms and clinical findings for differentiating pneumonia from bronchial infection, a reliable diagnosis of this condition requires a chest radiograph. Nevertheless, according to these recent data, our GPs seem to be much more inclined to give a rapid prescription of empirical antibiotics rather than to carry out a precise diagnosis of pneumonia. Various reasons could explain this near automatic use of antibiotics in LRTI in general medical practice. Firstly, busy physicians might prefer to prescribe antibiotics immediately rather than to start a complete investigation of the episode. Secondly, patients usually ask for antibiotics since they consider them the more expeditious way to eradicate their annoying respiratory symptoms.

The immediate consequence of excessive antibiotic prescription in LRTI is economic. This, however, would be of limited importance if, basically, only low-cost drugs were employed. Unfortunately, different factors, medical (antibiotic resistance, new pathogens) and particularly commercial (pressure from pharmaceutical companies to prescribe newly developed drugs), greatly favour the prescription of much more expensive alternatives and increase treatment costs substantially. More relevant to these immediate economic costs is the long-term development of antibiotic resistance related to excessive antibiotic prescription in LRTI and other common infections 
in the community. For example, pneumococcal susceptibility to tetracyclines and trimethoprim-sulphamethoxazole has substantially decreased since these antibiotics have been routinely prescribed for decades in LRTI. Haemophilus influenzae, by far the most common causative organism of infectious exacerbation of chronic bronchitis, today presents a significant level of resistance to aminopenicillins due to beta-lactamase production $[8,9]$. Pneumococcal penicillin-resistance, due to changes in the penicillin-binding proteins of the bacterial wall induced by genetic mutation, has rapidly disseminated because of a competitive selection of resistant strains, enhanced by the massive use of beta-lactamic agents in paediatric and adult respiratory infections [10-12]. Nevertheless, penicillin and aminopenicillins are still adequate treatments for pneumococcal respiratory infections, provided that they are administered in higher dosage [13].

More recent experiences also illustrate the long-term impact of antibiotic prescription patterns. Erythromycin and other macrolides became the first-line treatment for LRTI in Spain during the last decade, in part due to the high prevalence of Legionella pneumonia and Coxiella burnetii in several areas of Spain, and to the increasing rate of pencillin-resistant pneumococcal strains. Pneumococcal macrolide-resistance significantly increased in only 10 yrs [11]. These and other experiences clearly illustrate the longterm consequences of present therapeutic practices. The trend toward a wide prescription of third generation cephalosporins for community-acquired pneumonia and other LRTI, confirmed by these new data, appear to be particularly hazardous. There is experimental evidence that pneumococcal cephalosporin resistance could emerge much more rapidly than that to penicillin [14]. On the other hand, clinical evidence reporting a certain decrease of susceptibility to third generation cephalosporins by the pneumococcus is already being published [13]. All these facts clearly indicate the need for a much more prudent approach when prescribing antibiotics in LRTI.

What can be done to rationalize antibiotic prescription in LRTI in general medicine? The data provided by HUCHON et al. [3] suggest that, so far, GPs appear to be little influenced by national guidelines, and that in some countries attendance at postgraduate courses is very low. By contrast, GPs admitted being strongly influenced by pharmaceutical companies. Although specific guidelines have been published in some countries [15-17], they have focused mainly on pneumonia, showing little interest in bronchial infection. Furthermore, their dissemination was poor among generalists. Guidelines and postgraduate courses should play a central role in providing objective information to GPs, including key issues, such as: 1) defining which LRTI are most likely to benefit from antibiotic treatment; 2) encouraging the need for a specific diagnosis of pneumonia; 3 ) recommending the prescription of antibiotic regimens designed for each specific type of respiratory infection (acute bronchitis, exacerbation of chronic bronchitis and community-acquired pneumonia), taking into account the local patterns of antibiotic resistance, and specifically discouraging the use of unnecessary expensive antibiotics; 4) stating the importance of some preventative measures, such as influenza and pneumococcal vaccination, in specific groups of the population; and 5) educating the general population about the limitations of antibiotic treatments, the dangers when excessively prescribed, and the potential value of preventative measures.

\section{References}

1. Macfarlane JT, Colville A, Guion A, Macfarlane RM, Rose DH, Finch RG. Prospective study on aetiology and outcome of adult lower respiratory tract infections in the community. Lancet 1993; 341: 511-514.

2. Ortqvist A. Antibiotic treatment of community-acquired pneumonia in clinical practice: a European perspective. J Antimicrobial Chemother 1995; 35: 205-212.

3. Huchon GJ, Giraldoni Grassi G, Léophonte P, Manresa F, Schaberg T, Woodhead MA. Initial antibiotic therapy for adults with lower respiratory tract infection in the community: a European survey. Eur Respir J 1996; 9: $1590-1595$.

4. Woodhead M, Giraldoni Grassi G, Huchon GJ, Léophonte $P$, Manresa F, Schaberg T. Use of investigations in lower respiratory tract infection in the community: a European survey. Eur Respir J 1996; 9: 1596-1600.

5. Anthonisen NR, Manfreda J, Warren PV, Hershfield ES, Harding GKM, Nelson NA. Antibiotic therapy in exacerbations of chronic obstructive pulmonary disease. Ann Intern Med 1987; 106: 196-204.

6. Marrie TH, Durant H, Yates L. Community-acquired pneumonia requiring hospitalization: 5 year prospective study. Rev Infect Dis 1989; 11: 586-599.

7. British Thoracic Society and Public Health Laboratory Service. Community-acquired pneumonia in adults in British hospitals in 1982-1983: a survey of aetiology, mortality, prognostic factors and outcome. Q J Med 1987; 239: 195-220.

8. Needham CA. Haemophilus influenzae antibiotic susceptibility. Clin Microbiol Rev 1988; 1: 218-227.

9. Jorgensen JH, Doern GV, Mahler LA, Howell AW, Redding JS. Antimicrobiol resistance among respiratory isolates of Haemophilus influenzae: a global perspective. Antimicrob Agents Chemother 1990; 34: 2075-2080.

10. Marton A, Gulyas M, Muños R, Tomasz A. Extremely high incidence of antibiotic resistance of clinical isolates of Streptococcus pneumoniae in Hungary. J Infect Dis 1991; 163: 542-548.

11. Liñares J, Pallarés R, Alonso T, et al. Trends in antimicrobiol resistance of clinical isolates of Streptococcus pneumoniae in Bellvitge Hospital, Barcelona, Spain. Clin Infect Dis 1992; 15: 99-105.

12. Geslin P, Buu Hoi A, Frémaux A, Acar JF. Antimicrobial resistance in Streptococcus pneumoniae: an epidemiological survey in France 1970-1990. Clin Infect Dis 1992; 15: 95-98.

13. Pallares R, Liñares J, Vadillo $\mathrm{M}$, et al. Resistance to penicillin and cephalosporins and mortality from severe pneumococcal pneumonia in Barcelona, Spain. $N$ Engl J Med 1995; 33: 474-480.

14. Muñoz R, Dowson DG, Daniels M, et al. Genetics of resistance to third-generation cephalosporins in clinical isolates in Streptococcus pneumoniae. Mol Microbiol 1992; 17: 2641-2645.

15. Normativa SEPAR. Diagnóstico y tratamiento de las neumonías. Sociedad Española de Neumología y Cirugía Torácica. Barcelona, Ed Doyma, 1992.

16. British Thoracic Society. Guidelines for the management of community-acquired pneumonia in adults admitted to hospital. Br J Hosp Med 1993; 49: 346-350.

17. American Thoracic Society. Guidelines for the initial management of adults with community-acquired pneumonia: diagnosis, assessment of severity, and initial antimicrobial therapy. Am Rev Respir Dis 1993; 148: $1418-1426$. 